J. Austral. Math. Soc. 25 (Series A) (1978), 7-18

\title{
NIKOLSKII-TYPE INEQUALITIES FOR TRIGONOMETRIC POLYNOMIALS AND ENTIRE FUNCTIONS OF EXPONENTIAL TYPE
}

\author{
R. J. NESSEL and G. WILMES \\ (Received 13 April 1976) \\ Communicated by E. Strzelecki
}

\begin{abstract}
Nikolskii-type inequalities, thus inequalities between different metrics of a function, are established for trigonometric polynomials and $p$ th power integrable functions, $0<p \leqslant \infty$, of several variables having Fourier transform with compact support. It is shown that certain gaps in the spectra of the functions involved may be taken into account. As an immediate consequence it follows that the general results cover the classical inequalities which are concerned with functions of rectangular type. But at the same time one may give applications to functions of type $K$ where $K$ is a symmetric body in Euclidean $n$-space.
\end{abstract}

\section{Introduction}

Nikolskii-type inequalities, following the usual nomenclature, are, roughly speaking, inequalities between different metrics of the same function. Thus Nikolskii derived the inequalities $\dagger$ for $1 \leqslant p \leqslant q \leqslant \infty$ :

$$
\begin{gathered}
\left\|t_{m_{1}, \ldots, m_{n}}\right\|_{q, 2 \pi} \leqslant 2^{n}\left[(2 \pi)^{n} \prod_{j=1}^{n} m_{j}\right]^{1 / p-1 / q}\left\|t_{m_{1}, \ldots, m_{n}}\right\|_{p, 2 \pi}, \\
\left\|f_{\sigma_{1}, \ldots, \sigma_{n}}\right\|_{q} \leqslant 2^{n}\left[(2 \pi)^{n} \prod_{j=1}^{n} \sigma_{j}\right]^{1 / p-1 / q}\left\|f_{\sigma_{1}, \ldots, \sigma_{n}}\right\|_{p}
\end{gathered}
$$

for trigonometric polynomials of type

$$
t_{m_{1}, \ldots, m_{n}}:=\sum_{j_{1}=-m_{1}}^{m_{1}} \ldots \sum_{j_{n}=-m_{n}}^{m_{n}} c_{j_{1}, \ldots, j_{n}} \exp \left(i\left(j_{1} x_{1}+\ldots+j_{n} x_{n}\right)\right)
$$

as well as for entire functions $f_{\sigma_{1}, \ldots, \sigma_{n}}\left(z_{1}, \ldots, z_{n}\right)$ of exponential (rectangular, see (4.11)) type $\sigma_{1}, \ldots, \sigma_{n}>0$, respectively (see Nikolskii, 1951, and 1974, p. 126). For (one-dimensional trigonometric) polynomials analogous results were obtained by

The contribution of this author was supported by Grant No. II B7-FA 5334 awarded by the Minister für Wissenschaft und Forschung des Landes Nordrhein-Westfalen.

t Our notations $\|\cdot\|_{p, 2 \pi},\|\cdot\|_{p}$ (see (2.1), (3.1)) differ from those in Nikolskii $(1951,1974)$ by a factor $(2 \pi)^{n / p}$. 
Szegö and Zygmund (1953) (see also Zygmund (1959, I, p. 154)), and in the particular case $q=\infty$ already by Jackson (1933) (see also Achieser, 1967, p. 178).

In numerous papers (see Ibragimov $(1958,1959,1965)$; Ibragimov and Dzafarov (1961); Ibragimov and Mamedhanov (1964); Ibragimov and Rymarenko (1966); Mamedhanov (1964); Sabziev (1965); Timan (1963, pp. 227-236)) (1.1) and (1.2) are sharpened and extended. In particular, the inequality

$$
\left\|f_{\sigma_{1}, \ldots, \sigma_{n}}\right\|_{q} \leqslant\left[\left(2 p_{0}\right)^{n} \prod_{j=1}^{n} \sigma_{j}\right]^{1 / p-1 / q}\left\|f_{\sigma_{1}, \ldots, \sigma_{n}}\right\|_{p}
$$

$p_{0}$ denoting the smallest integer not less than $p / 2$, is derived in Ibragimov (1959), Ibragimov and Dzafarov (1961) and Timan (1963). The corresponding result

$$
\left\|t_{m_{1}, \ldots, m_{n}}\right\|_{q, 2 \pi} \leqslant\left[\prod_{j=1}^{n}\left(2 p_{0} m_{j}+1\right)\right]^{1 / p-1 / q}\left\|t_{m_{1}, \ldots, m_{n}}\right\|_{p, 2 \pi}
$$

for trigonometric polynomials of type (1.3) is developed in Ibragimov (1958), Timan (1963) and Sabziev (1965). The constants in (1.4), (1.5) seem to be the best known so far, their optimal values being an open problem. Inequalities with respect to norms on subintervals are established in Bari (1954). For functions which are positive for real values of their arguments, (1.4) is sharpened still further in Mamedhanov (1964). Analogous inequalities for weighted metrics and in generalized Lebesgue spaces are considered in Ibragimov (1965), Ibragimov and Dzafarov (1961), Ibragimov and Mamedhanov (1964), Ibragimov and Rymarenko (1966), Mamedhanov (1964), Sabziev (1965), Triebel (1977). For similar estimates concerning algebraic polynomials of (real) variables (on compact sets) see Ganzburg (1975), Lebed (1957), Szegö and Zygmund (1953), Timan (1963, p. 235). For inequalities concerning polynomials of a complex variable on curves see Mamedhanov (1974). Moreover, Nikolskii-type inequalities in connection with orthonormal systems of functions are treated in Jackson (1933), Nessel and Wilmes (1976), Nessel and Wilmes (to appear), Timan (1975), Watari and Okuyama (1975). For further details and related topics one may also consult Burenkov (1968), Görlich and Paulus (to appear), and the literature cited there.

The purpose of this note is to observe that in inequalities of the above type one may in fact take into account certain gaps in the spectrum of the function involved. Indeed, it follows in Section 2 that the ratio $\|t\|_{q, 2 \pi} /\|t\|_{p, 2 \pi}$ essentially depends upon the number of nonzero coefficients and not upon a specific definition of the degree of the trigonometric polynomial $t$. In Section 3 corresponding estimates are established for $L^{p}\left(\mathbf{R}^{n}\right)$-functions having Fourier transform with compact support. The theorem of Paley-Wiener-Schwartz is then used in Section 4 to derive inequalities for entire functions of exponential type $K$, where $K$ is a symmetric body. As an immediate application of Theorems 2 and 5 the inequalities (1.4) and (1.5) (including constants) are regained as special cases. 
The authors express their sincere gratitude to Professor W. Hayman, London, for his valuable advice in connection with the case $0<p<1$ of Theorem 5 as well as to Professor E. Görlich and G. Paulus for various hints concerning the literature and for a critical reading of the manuscript. For further stimulating discussions the authors would like to thank the participants of the colloquium lectures held by the first-named author at Delft, Eindhoven, and Leiden in the week of 22-25 March 1976.

\section{Inequalities for trigonometric polynomials}

Let $\mathbf{R}^{n}$ be the Euclidean $n$-space with elements $x:=\left(x_{1}, \ldots, x_{n}\right), y, v, \ldots$, inner product $x y:=\sum_{j=1}^{n} x_{j} y_{j}$, and norm $|x|:=\sqrt{ }(x x)$. Let $\mathbf{Q}^{n} \subset \mathbf{R}^{n}$ be the cube $\left\{x \in \mathbf{R}^{n}\right.$; $\left.-\pi \leqslant x_{j}<\pi, 1 \leqslant j \leqslant n\right\}$ and $L_{2 \pi}^{p}, 0<p \leqslant \infty$, the space of measurable functions with period $2 \pi$ in each variable for which

$$
\|f\|_{p, 2 \pi}:=\left\{(2 \pi)^{-n} \cdot \int_{\mathbf{Q}^{n}}|f(u)|^{p} d u\right\}^{1 / p},\|f\|_{\infty}:=\underset{u \in \mathbf{Q}^{n}}{\operatorname{ess} s u p}|f(u)|,
$$

respectively, is finite. $Z^{n}$ denotes the set of all integral lattice points $k$ in $\mathbf{R}^{n}$. For $f, g \in L_{2 \pi}^{1}$ convolution and $k$ th Fourier coefficient are given by

$$
(f * g)(x):=(2 \pi)^{-n} \int_{Q^{n}} f(x-u) g(u) d u, f^{\wedge}(k):=(2 \pi)^{-n} \int_{Q^{n}} f(u) \exp (-i k u) d u .
$$

Let an arbitrary trigonometric polynomial be given via

$$
t(x):=\sum_{k \in \operatorname{supp} \iota^{\wedge}} c_{k} \exp (i k x), \quad \operatorname{supp} t^{\wedge}:=\left\{k \in \mathbf{Z}^{n} ; t^{\wedge}(k)=c_{k} \neq 0\right\},
$$

and let $\mathfrak{N}(G)$ denote the number of lattice points in $G \subset \mathbf{R}^{n}$, in other words, $\mathfrak{N}\left(\operatorname{supp} t^{\wedge}\right)$ is the number of nonzero coefficients of the polynomial $t$.

THEOREM 1. For each trigonometric polynomial (2.2) there holds the inequality $(1 \leqslant p \leqslant 2, p \leqslant q \leqslant \infty)$

$$
\|t\|_{q, 2 \pi} \leqslant \mathfrak{N}\left(\operatorname{supp} t^{\wedge}\right)^{1 / p-1 / q}\|t\|_{p, 2 \pi} .
$$

ProOF. If $D(x):=\sum_{k \in \operatorname{supp} \leadsto} \exp (i k x)$ denotes the corresponding Dirichlet kernel, the Hausdorff-Young inequality yields for any $r \geqslant 2,1 / r+1 / r^{\prime}=1$,

$$
\|D\|_{r, 2 \pi} \leqslant\left\|D^{\wedge}(k)\right\|_{r^{r}}:=\left\{\sum_{k \in \operatorname{supp} \ell^{\wedge}} \mid D^{\wedge}(k) r^{r^{\prime}}\right\}^{1 / r^{\prime}}=\mathfrak{N}\left(\operatorname{supp} t^{\wedge}\right)^{1 / r^{\prime}} .
$$

Hence by the convolution theorem and Young's inequality one has

$$
\|t\|_{q, 2 \pi}=\|D * t\|_{q, 2 \pi} \leqslant\|t\|_{p, 2 \pi}\|D\|_{r, 2 \pi} \leqslant \mathfrak{N}\left(\operatorname{supp} t^{\wedge}\right)^{1 / p-1 / q}\|t\|_{p, 2 \pi}
$$

for all values $1 \leqslant p<q \leqslant \infty$ satisfying $1 / p-1 / q=1-1 / r=1 / r^{\prime} \geqslant \frac{1}{2}$. In particular, 
$\|t\|_{\infty, 2 \pi} \leqslant \mathfrak{N}\left(\operatorname{supp} t^{\wedge}\right)^{1 / p}\|t\|_{p, 2 \pi}$ for all $1 \leqslant p \leqslant 2$, which implies (2.3) since $\|t\|_{q, 2 \pi}$ is a logarithmically convex function of $1 / q$ (see Zygmund (1959, I, p. 25)).

Let us point out that the factor in the inequality (2.3) only depends upon the number of nonzero coefficients and not upon a specific definition of the degree of the polynomial in question. For example, if in case $n=1$ we take $t_{0}(x):=\exp (\operatorname{im} x)$ for some $m \in \mathbf{Z}$, then (2.3) (together with (2.4)) reproduces the triviality $\left\|t_{0}\right\|_{q, 2 \pi} \leqslant\left\|t_{0}\right\|_{p, 2 \pi}$ (for all $0<p \leqslant q \leqslant \infty$ ) whereas (1.5) would yield

$$
\left\|t_{0}\right\|_{q, 2 \pi} \leqslant\left(2 p_{0}|m|+1\right)^{1 / p-1 / q}\left\|t_{0}\right\|_{p, 2 \pi} .
$$

This observation may be of some interest in studying lacunary series. Moreover, the example of the Dirichlet kernel shows that the constant in (2.3) is the best possible one for $p=2, q=\infty$. Note, however, that (2.3) is in general not true for $p>2$. For, otherwise, the example of the Dirichlet kernel would lead to $\left\|\sum_{j=-m}^{m} \exp (i j x)\right\|_{p}=(2 m+1)^{1 / p^{\prime}}$, a contradiction to an extremal property of the Hausdorff-Young inequality (see Zygmund (1959, II, p. 105)).

To derive inequalities of type (2.3) for arbitrary $p>0$, we have to restrict ourselves to convex spectra.

THEOREM 2. For each trigonometric polynomial (2.2) there holds the inequality $(0<p \leqslant q \leqslant \infty)$

$$
\|t\|_{q, 2 \pi} \leqslant \mathfrak{N}\left[p_{0} \operatorname{conv}\left(\operatorname{supp} t^{\wedge}\right)\right]^{1 / p-1 / q}\|t\|_{p, 2 \pi},
$$

where $p_{0}$ is the smallest integer not less than $p / 2$, and $\operatorname{conv}\left(\operatorname{supp} t^{\wedge}\right)$ denotes the convex hull of supp $t^{\wedge}$.

Proof. Following a device in Timan (1963, p. 229), consider the polynomial $t^{p_{0}}$. Then

$$
\operatorname{supp}\left(t^{p_{0}}\right)^{\wedge} \subset \operatorname{supp} t^{\wedge}+\ldots+\operatorname{supp} t^{\wedge} \subset p_{0} \operatorname{conv}\left(\operatorname{supp} t^{\wedge}\right),
$$

and by Theorem 1 one has

$$
\begin{aligned}
\|t\|_{\infty, 2 \pi}^{p_{0}}=\left\|t^{p_{0}}\right\|_{\infty, 2 \pi} & \leqslant \mathfrak{N}\left[\operatorname{supp}\left(t^{p_{0}}\right)^{\wedge}\right]^{\xi}\left\|t^{p_{0}}\right\|_{2,2 \pi} \\
& \leqslant \mathfrak{N}\left[p_{0} \operatorname{conv}\left(\operatorname{supp} t^{\wedge}\right)\right]^{\}}\|t\|_{\infty, 2 \pi}^{p_{0}-p / 2}\|t\|_{p, 2 \pi}^{p / 2},
\end{aligned}
$$

which implies (2.4) for $q=\infty$. From this the assertion follows as in the proof of Theorem 1.

The proof indeed shows that for $0<p<1$ even the assertion (2.3) remains valid. Moreover, one may replace $\left[p_{0} \operatorname{conv}\left(\operatorname{supp} t^{\wedge}\right)\right]$ in $(2.4)$ by $\left[\operatorname{supp} t^{\wedge}+\ldots+\operatorname{supp} t^{\wedge}\right]$.

Note that (2.4) not only covers (1.5) since

$$
p_{0} \operatorname{conv}\left(\operatorname{supp} t_{m_{1}, \ldots, m_{n}}^{\hat{n}}\right) \subset\left\{x \in \mathbf{R}^{n} ;\left|x_{j}\right| \leqslant p_{0} m_{j}, 1 \leqslant j \leqslant n\right\},
$$


but also delivers inequalities with respect to other classical definitions of the degree of trigonometric polynomials of several variables. For the radial case compare Corollary 2 in connection with classical results of the geometry of numbers.

\section{Inequalities for functions with compact spectra}

Let $L^{p}\left(\mathbf{R}^{n}\right), 0<p \leqslant \infty$, be the space of measurable functions for which

$$
\|f\|_{p}:=\left\{(2 \pi)^{-n} \int_{\mathbf{R}^{n}}|f(u)|^{p} d u\right\}^{1 / p},\|f\|_{\infty}:=\underset{u \in \mathbf{R}^{n}}{\operatorname{ess} \sup }|f(u)|,
$$

respectively, is finite. Let $\mathfrak{S}\left(\mathbf{R}^{n}\right)$ be the Schwartzian space of infinitely differentiable functions, rapidly decreasing at infinity, and $\widetilde{S}^{\prime}$ the corresponding dual space of tempered distributions. The Fourier transform of $f \in \mathfrak{S}^{\prime}$ is given by

$$
f^{\wedge}(\varphi):=f\left(\varphi^{\wedge}\right), \quad \varphi^{\wedge}(v):=(2 \pi)^{-n} \int_{\mathbb{R}^{n}} \varphi(x) \exp (-i v x) d x \quad(\varphi \in \mathbb{S}) .
$$

Theorem 3. Let $f \in L^{p}\left(\mathbf{R}^{n}\right), 1 \leqslant p \leqslant 2$, be such that $\operatorname{supp} f^{\wedge}$ is compact. Then $f$ belongs to $L^{q}\left(\mathbf{R}^{n}\right)$ for all $p \leqslant q \leqslant \infty$ and satisfies

$$
\|f\|_{q} \leqslant\left[\text { meas }\left(\operatorname{supp} f^{\wedge}\right)\right]^{1 / p-1 / q}\|f\|_{p},
$$

where meas $\left(\operatorname{supp} f^{\wedge}\right)$ denotes the Lebesgue measure of the support of $f^{\wedge}$. Again the constant in (3.2) is the best possible one in case $p=2, q=\infty$.

ProOF. Let $\kappa$ denote the characteristic function of $\operatorname{supp} f^{\wedge}$, and let $\psi \in L^{2}\left(\mathbf{R}^{n}\right)$ be such that $\psi^{\wedge}=\kappa$, thus $\psi(x)=(2 \pi)^{n} \kappa^{\wedge}(-x)$. Since $\kappa \in L^{1}\left(\mathbf{R}^{n}\right) \cap L^{2}\left(\mathbf{R}^{n}\right)$, Titchmarsh's inequality implies $\psi \in L^{r}\left(\mathbf{R}^{n}\right)$ for each $2 \leqslant r \leqslant \infty$ and $\left(1 / r+1 / r^{\prime}=1\right)$

$$
\|\psi\|_{r}=\left\|(2 \pi)^{n} \kappa^{\wedge}\right\|_{r} \leqslant(2 \pi)^{n / r^{\prime}}\|\kappa\|_{r^{\prime}}=\left[\operatorname{meas}\left(\operatorname{supp} f^{\wedge}\right)\right]^{1 / r^{\prime}} .
$$

Moreover, $f^{\wedge}=f^{\wedge} \kappa \in L^{1}\left(\mathbf{R}^{n}\right)$, and hence by the Parseval formula (see Butzer and Nessel (1971, p. 212))

$$
(2 \pi)^{n}\left(f^{\wedge} \kappa\right)^{\wedge}(-x)=(f * \psi)(x):=(2 \pi)^{-n} \int_{\mathbf{R}^{n}} f(x-u) \psi(u) d u .
$$

Therefore $f * \psi=f$, and Young's inequality yields

$$
\|f\|_{q} \leqslant\|\psi\|_{r}\|f\|_{p} \leqslant\left[\operatorname{meas}\left(\operatorname{supp} f^{\wedge}\right)\right]^{1 / r^{\prime}}\|f\|_{p}
$$

for any $1 / r^{\prime}=1 / p-1 / q \geqslant \frac{1}{2}$. In particular, for $1 \leqslant p \leqslant 2$ one has

$$
\|f\|_{\infty} \leqslant\left[\operatorname{meas}\left(\operatorname{supp} f^{\wedge}\right)\right]^{1 / p}\|f\|_{p} .
$$

Hence $f \in L^{p}\left(\mathbf{R}^{n}\right) \cap L^{\infty}\left(\mathbf{R}^{n}\right)$, and the logarithmic convexity of the norm then implies (3.2) as in the previous section. The example of the function $\psi$ shows that the constant in (3.2) is best possible in case $p=2, q=\infty$. 
To establish a Nikolskii inequality for arbitrary values of $p$ we proceed as in the trigonometric case.

THEOREM 4. Let $f \in L^{p}\left(\mathbf{R}^{n}\right), 1 \leqslant p \leqslant \infty$, be such that $\operatorname{supp} f^{\wedge}$ is compact. Then $f$ belongs to $L^{q}\left(\mathbf{R}^{n}\right)$ for all $p \leqslant q \leqslant \infty$ and satisfies

$$
\|f\|_{q} \leqslant\left\{p_{0}^{n} \text { meas }\left[\operatorname{conv}\left(\operatorname{supp} f^{\wedge}\right)\right]\right\}^{1 / p-1 / q}\|f\|_{p}
$$

with $p_{0}$ as in Theorem 2. The assertion remains valid for $0<p<1$ (and then even in the form (3.2)) if one additionally assumes $f \in \Xi^{\prime}\left(\mathbf{R}^{n}\right)$.

Proof. Let $1 \leqslant p \leqslant \infty$. Let $\psi \in \mathfrak{S}\left(\mathbf{R}^{n}\right)$ be such that $\psi^{\wedge}$ equals 1 in a compact neighbourhood of $\operatorname{supp} f^{\wedge}$. Then $f=f * \psi$, so that $f$ is bounded and

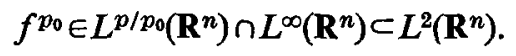

Moreover (see Treves, $(1967$, p. 314) $)\left(f^{p_{0}}\right)^{\wedge}$ is equal to the $p_{0}$-fold convolution product $f^{\wedge} * \ldots * f^{\wedge}$, and thus one has (see Friedman (1963, p. 77))

$$
\operatorname{supp}\left(f^{p_{0}}\right)^{\wedge} \subset \operatorname{supp} f^{\wedge}+\ldots+\operatorname{supp} f^{\wedge} \subset p_{0} \operatorname{conv}\left(\operatorname{supp} f^{\wedge}\right) .
$$

Since meas $\left[p_{0} \operatorname{conv}\left(\operatorname{supp} f^{\wedge}\right)\right]=p_{0}^{n}$ meas $\left[\operatorname{conv}\left(\operatorname{supp} f^{\wedge}\right)\right]$, (3.3) may then be derived from Theorem 3 as in the trigonometric case.

In the case $0<p<1$ it now suffices to show that the present assumptions imply $f \in L^{\infty}\left(\mathbf{R}^{n}\right)$. Indeed, let $f \in L^{p}\left(\mathbf{R}^{n}\right) \cap \mathcal{S}^{\prime}\left(\mathbf{R}^{n}\right)$ be such that $\operatorname{supp} f^{\wedge}$ is compact. Then, for example by the theorem of Paley-Wiener-Schwartz (see Section 4), this implies in particular that $f$ is continuous. Thus, choosing $\psi_{\epsilon}(x):=\delta^{\wedge}(-\varepsilon x), \varepsilon>0$, where $\delta \in \subseteq\left(\mathbf{R}^{n}\right)$ is positive satisfying

$$
(2 \pi)^{-n} \int_{\mathbf{R}^{n}} \delta(u) d u=1, \quad \operatorname{supp} \delta \subset\left\{u \in \mathbf{R}^{n} ;|u| \leqslant 1\right\},
$$

one has $\left(f \psi_{\varepsilon}\right)^{\wedge} \approx f^{\wedge} * \varepsilon^{-n} \delta(. / \varepsilon) \in \mathbb{S}\left(\mathbf{R}^{n}\right)$ and $\lim _{\varepsilon \rightarrow 0+}\left(f \psi_{\varepsilon}\right)(x)=f(x)$ for all $x \in \mathbf{R}^{n}$. Moreover, since $f \psi_{\varepsilon} \in L^{\infty}\left(\mathbf{R}^{n}\right)$ and $\operatorname{supp}\left(f \psi_{\varepsilon}\right)^{\wedge} \subset \operatorname{supp} f^{\wedge}+\operatorname{supp} \delta$ uniformly for all $0<\varepsilon<1$, one may conclude as in the previous cases that

$$
\left\|f \psi_{\epsilon}\right\|_{\infty} \leqslant \text { const }\left\|f \psi_{\epsilon}\right\|_{p} \leqslant \text { const }\left\|\psi_{\epsilon}\right\|_{\infty}\|f\|_{p}=\text { const }\|f\|_{p} .
$$

Thus one has for all $x \in \mathbf{R}^{n}$

$$
|f(x)| \leqslant\left|f(x)-\left(f \psi_{\epsilon}\right)(x)\right|+\left\|f \psi_{\epsilon}\right\|_{\infty} \leqslant \text { const }\|f\|_{p}+o(1) \quad(\varepsilon \rightarrow 0+)
$$

which shows that $f$ is bounded.

Let us mention that (3.3) again gives an upper bound for the ratio $\|f\|_{q} /\|f\|_{p}$ which only depends upon the measure of the convex hull of the support of $f^{\wedge}$ (and not, for example, upon the symmetric (to the origin) convex hull; but see Section 4). 


\section{Inequalities for entire functions of exponential type $K$}

In this section we would like to show that the results of the previous section cover (1.4), at the same time generalizing it from rectangular type to a rather general notion of exponential type.

Following Stein and Weiss (1971, pp. 111ff.), let $K \subset \mathbf{R}^{n}$ be a symmetric body, thus a compact, convex, symmetric $(x \in K$ implies $-x \in K)$ subset of $\mathbf{R}^{n}$ with nonempty interior, and let $K^{*}:=\left\{y \in \mathbf{R}^{n} ;|x y| \leqslant 1\right.$ for all $\left.x \in K\right\}$ be its polar set. Note that $K$ is a symmetric body if and only if it is the unit sphere with respect to a norm $\|\cdot\|_{K}$ on $\mathbf{R}^{n}$. Furthermore, $K^{*}$ is again a symmetric body, and one has

$$
\begin{aligned}
& \left(K^{*}\right)^{*}=K, \\
& \|x\|_{K}=\sup _{y \in K^{*}}|x y|, \quad\|x\|_{K^{*}}=\sup _{y \in K}|x y|, \\
& (\alpha K)^{*}=\alpha^{-1} K^{*}, \quad\|x\|_{\alpha K}=\alpha^{-1}\|x\|_{K} \quad(\alpha>0) .
\end{aligned}
$$

Let $\mathbf{C}^{n}$ be the $n$-fold Cartesian product of the complex plane $\mathbf{C}$ with elements $z:=\left(z_{1}, \ldots, z_{n}\right):=u+i v$ (with $\left.u, v \in \mathbf{R}^{n}\right)$ and norm $|z|:=\left(\sum_{j=1}^{n}\left|z_{j}\right|^{2}\right)^{\frac{1}{2}}$. Then (4.2) can be extended to a norm on $\mathbf{C}^{n}$ by setting

$$
\|z\|_{\mathbb{K}^{*}}:=\sup _{\boldsymbol{y} \in \mathbb{K}^{*}}|z y|=\sup _{y \in \mathbb{K}^{*}}|u y+i v y| \text {. }
$$

An entire function $F$, defined on $\mathbf{C}^{n}$, is said to be of exponential type $K$ if for each $\varepsilon>0$ there exists a constant $A_{\varepsilon}>0$ such that (see Stein and Weiss (1971, p. 112))

$$
|F(z)| \leqslant A_{\epsilon} \exp \left\{(1+\varepsilon)\|z\|_{\boldsymbol{K}}\right\} \quad\left(z \in \mathbf{C}^{n}\right) .
$$

Let $\mathbb{E}(K)$ be the set of all entire functions of exponential type $K$, and let $\mathfrak{E}_{p}(K)$, $0<p \leqslant \infty$, be the subset of those functions $f \in \mathbb{E}(K)$ whose restriction to $\mathbf{R}^{n}$ belongs to $L^{p}\left(\mathbf{R}^{n}\right)$. In this case, if no confusion may occur, the same notation is applied to $f \in \mathfrak{E}_{p}(K)$ and its restriction to $\mathbf{R}^{n}$. In this terminology, one has

Theorem of Paley-Wiener-Schwartz. Let $K \subset \mathbf{R}^{n}$ be a symmetric body and $f \in S^{\prime}$. The following assertions are equivalent:

(i) $f^{\wedge}$ has support in $K^{*}$.

(ii) $f$ can be extended to $\mathbf{C}^{n}$ as an entire function $f(z)$ such that there is an integer $m \geqslant 0$ and $a$ constant $C \geqslant 0$ such that for all $z=u+i v$

$$
|f(z)| \leqslant C(1+|z|)^{m} \exp \left\{\|v\|_{\mathcal{K}}\right\} .
$$

(iii) $f$ can be extended to a function in $\mathfrak{E}(K)$.

An explicit proof of the equivalence of (i) and (ii) is given in Treves (1967, pp. 311-312). For functions in $L^{2}\left(\mathbf{R}^{n}\right)$ the equivalence of (i) and (iii) is proved in Stein and Weiss (1971, pp. 112-114). The extension to general tempered distributions may be performed analogously to the procedure described in Friedman 
(1963, p. 145) and Schwartz (1966, p. 272), where the above result is proved for rectangles $K$.

THEOREM 5. Let $K$ be a symmetric body. If $f \in \mathfrak{E}_{p}(K)$ for some $0<p \leqslant \infty$, then f belongs to $\mathfrak{E}_{q}(K)$ for all $p \leqslant q \leqslant \infty$ and satisfies

$$
\|f\|_{q} \leqslant\left[p_{0}^{n} \text { meas } K^{*}\right]^{1 / p-1 / q}\|f\|_{p} .
$$

Proof. In view of the Theorem of Paley-Wiener-Schwartz and Theorem 4 it only remains to show that $f \in \mathfrak{E}_{p}(K), 0<p<1$, implies $f \in L^{\infty}\left(\mathbf{R}^{n}\right)$. For $n=1$ and $K=[-a, a] \subset \mathbf{R}, a>0$, this result is proved in Boas $(1954, p .98)$. For the sake of completeness let us reduce the case $n>1$ to the one-dimensional result via induction, using the theorem of Paley-Wiener-Schwartz and the Nikolskii inequality of Theorem 4.

Let $n \in \mathbf{N}$ be fixed and assume

$$
\mathfrak{E}_{p}\left(K^{\prime}\right) \subset \mathfrak{E}_{\infty}\left(K^{\prime}\right) \quad(0<p<1)
$$

for all symmetric bodies $K^{\prime} \subset \mathbf{R}^{m}, 1 \leqslant m \leqslant n$. Let $K \subset \mathbf{R}^{n+1}$ be a symmetric body, and let $f \in \mathfrak{E}_{p}(K)$. It follows from Fubini's theorem that

$$
\begin{aligned}
h\left(x_{n+1}\right) & :=\int_{\mathbf{R}^{n}}\left|f\left(x, x_{n+1}\right)\right|^{p} d x \in L^{1}(\mathbf{R}), \\
g(x) & :=\int_{\mathbf{R}}\left|f\left(x, x_{n+1}\right)\right|^{p} d x_{n+1} \in L^{1}\left(\mathbf{R}^{n}\right) .
\end{aligned}
$$

Since all norms on a finite dimensional space are equivalent, one can choose symmetric bodies $K^{\prime} \subset \mathbf{R}^{n}$ and $K^{\prime \prime} \subset \mathbf{R}^{1}$ such that $\|z\|_{K} \leqslant\left\|z^{\prime}\right\|_{K^{\prime}}+\left\|z_{n+1}\right\|_{K^{\prime \prime}}$ for all $\left(z^{\prime}, z_{n+1}\right):=z \in \mathbf{C}^{n+1}$. Then it follows from (4.7) that $f\left(z^{\prime}, x_{n+1}\right)$ considered as a function of $z^{\prime} \in \mathbf{C}^{n}$ belongs to $\mathfrak{E}_{p}\left(K^{\prime}\right)$ for at least almost all $x_{n+1} \in \mathbf{R}$. Analogously one has $f\left(x, z_{n+1}\right) \in \mathbb{E}_{p}\left(K^{\prime \prime}\right)$ for almost all $x \in \mathbf{R}^{n}$. Since

$$
\mathfrak{E}_{p}\left(K^{\prime}\right) \subset \mathfrak{E}_{\infty}\left(K^{\prime}\right), \quad \mathfrak{E}_{p}\left(K^{\prime \prime}\right) \subset \mathfrak{E}_{\infty}\left(K^{\prime \prime}\right)
$$

by assumption, one may apply the Theorem of Paley-Wiener-Schwartz and Theorem 4 to conclude (see (4.7), (4.8))

$$
\begin{aligned}
& \sup _{x \in \mathbf{R}^{n}}\left|f\left(x, x_{n+1}\right)\right| \leqslant\left[\text { meas } K^{\prime *}\right]^{1 / p}\left|h\left(x_{n+1}\right)\right|^{1 / p} \quad \text { almost everywhere in } \mathbf{R}, \\
& \sup _{x_{n+1} \in \mathbf{R}}\left|f\left(x, x_{n+1}\right)\right| \leqslant\left[\text { meas } K^{\prime \prime *}\right]^{1 / p}|g(x)|^{1 / p} \quad \text { almost everywhere in } \mathbf{R}^{n} .
\end{aligned}
$$

Thus one has for almost all $\left(x, x_{n+1}\right) \in \mathbf{R}^{n+1}$

$$
\left|f\left(x, x_{n+1}\right)\right|^{2 p} \leqslant \text { const }|g(x)|\left|h\left(x_{n+1}\right)\right| \in L^{1}\left(\mathbf{R}^{n+1}\right),
$$


and hence $f \in \mathfrak{E}_{2 p}(K)$. An appropriate iteration of the above argument yields $f_{\in} \mathfrak{E}_{q}(K)$ for some $1 \leqslant q$ which implies $f \in \mathfrak{E}_{\infty}(K)$ by Theorem 4 in connection with the Theorem of Paley-Wiener-Schwartz.

Let us consider two special cases of symmetric bodies, namely (see Stein and Weiss $(1971$, p. 111))

$$
K_{1}:=\left\{x \in \mathbf{R}^{n} ; \sigma_{1}\left|x_{1}\right|+\ldots+\sigma_{n}\left|x_{n}\right| \leqslant 1\right\}, \quad K_{2}:=\left\{x \in \mathbf{R}^{n} ;|x| \leqslant a^{-1}\right\}
$$

for some $\sigma_{1}, \ldots, \sigma_{n}>0$ and $a>0$, respectively. It is easy to check that for all $x \in \mathbf{R}^{n}$

$$
\begin{aligned}
& K_{1}^{*}=\left\{y \in \mathbf{R}^{n} ;\left|y_{j}\right| \leqslant \sigma_{j}, 1 \leqslant j \leqslant n\right\},\|x\|_{K_{1}}=\sum_{j=1}^{n} \sigma_{j}\left|x_{j}\right|, \\
& K_{2}^{*}=\left\{y \in \mathbf{R}^{n} ;|y| \leqslant a\right\},\|x\|_{K_{2}}=a|x| .
\end{aligned}
$$

However, for $z \in \mathbf{C}^{n}$ one only has

$$
\|z\|_{K_{1}} \leqslant \sum_{j=1}^{n} \sigma_{j}\left|z_{j}\right|, \quad\|z\|_{K_{\mathbf{2}}} \leqslant a|z| .
$$

Even though equality does not hold for general $z \in \mathbf{C}^{n}$, the sets $\mathfrak{F}_{\infty}\left(K_{1}\right), \mathfrak{E}_{\infty}\left(K_{2}\right)$ consist precisely of those entire functions $f$ whose restrictions to $\mathbf{R}^{n}$ are bounded and which are of rectangular type $\sigma_{1}, \ldots, \sigma_{n}>0$, thus

$$
|f(z)| \leqslant A_{\epsilon} \exp \left\{\sum_{j=1}^{n}\left(\sigma_{j}+\varepsilon\right)\left|z_{j}\right|\right\} \quad\left(z \in \mathbf{C}^{n}, \varepsilon>0\right),
$$

or of radial type $a>0$, thus

$$
|f(z)| \leqslant A_{6} \exp \{(a+\varepsilon)|z|\} \quad\left(z \in \mathbf{C}^{n}, \varepsilon>0\right),
$$

respectively. This is a consequence of the following

LEMMA. Let $\|\cdot\|$ be an arbitrary norm on $\mathbf{C}^{n}$, and let $f$ be an entire function such that for each $\varepsilon>0$ one has $|f(z)| \leqslant A_{\varepsilon} \exp \{(1+\varepsilon)\|z\|\}$ for all $z=u+i v \in \mathbf{C}^{n}$. If the restriction of $f$ to $\mathbf{R}^{n}$ is bounded, then $f$ satisfies

$$
|f(u+i v)| \leqslant\|f\|_{\infty} \exp \{\|v\|\} .
$$

For $n=1$ this result is proved in Stein and Weiss (1971, p. 109), to which (4.13) may be reduced by an argument as given for $p=2$ (instead of $p=\infty$ ) in Stein and Weiss (1971, p. 113). Observing that

$$
\text { meas } K_{1}^{*}=2^{n} \prod_{j=1}^{n} \sigma_{j}, \quad \text { meas } K_{2}^{*}=\frac{2 \pi^{\frac{3}{n}}}{n \Gamma\left(\frac{1}{2} n\right)} a^{n},
$$

one may state the following inequalities. 
COROllaRY 1. Let $f \in L^{p}\left(\mathbf{R}^{n}\right), 0<p \leqslant \infty$, be the restriction to $\mathbf{R}^{n}$ of an entire function of rectangular type $\sigma_{1}, \ldots, \sigma_{n}$ for some $\sigma_{j}>0,1 \leqslant j \leqslant n$. Then $f$ belongs to $L^{q}\left(\mathbf{R}^{n}\right)$ for all $p \leqslant q \leqslant \infty$ and

$$
\|f\|_{q} \leqslant\left[\left(2 p_{0}\right)^{n} \prod_{j=1}^{n} \sigma_{j}\right]^{1 / p-1 / q}\|f\|_{p} .
$$

Indeed, the assumptions again imply $f \in L^{\infty}\left(\mathbf{R}^{n}\right)$. Therefore by (4.9) and the lemma

$$
|f(u+i v)| \leqslant\|f\|_{\infty} \exp \left\{\sum_{j=1}^{n} \sigma_{j}\left|v_{j}\right|\right\}=\|f\|_{\infty} \exp \left\{\|v\|_{\mathcal{K}_{1}}\right\},
$$

and thus $f \in \mathcal{F}_{p}\left(K_{1}\right)$ so that the assertion follows by Theorem 5 . Note that (4.14) regains (1.4) including constants.

Corollary 2. Let $f \in L^{p}\left(\mathbf{R}^{n}\right), 0<p \leqslant \infty$, be the restriction to $\mathbf{R}^{n}$ of an entire function of radial type $a>0$. Then f belongs to $L^{q}\left(\mathbf{R}^{n}\right)$ for all $p \leqslant q \leqslant \infty$ and

$$
\|f\|_{q} \leqslant\left[\frac{2 \pi^{\frac{1}{2} n}}{n \Gamma\left(\frac{1}{2} n\right)}\left(p_{0} a\right)^{n}\right]^{1 / p-1 / q}\|f\|_{p} .
$$

For various applications of Nikolskii-type inequalities one may consult Burenkov (1968), Jackson (1933), Mertens et al. (1976), Nessel and Wilmes (to appear), Nikolskii (1951, 1974), Szalay (1974), Timan (1963, p. 365), Triebel (1977), Watari and Okuyama (1975).

\section{References}

N. I. Achieser (1967), Vorlesungen über Approximationstheorie (Akademie Verlag, Berlin, 1967).

N. K. Bari (1954), "A generalization of inequalities of S. N. Bernstein and A. A. Markov", Izv. Akad. Nauk SSSR Ser. Mat. 18, 59-176 (Russian).

R. P. Boas (1954), Entire Functions (Academic Press, New York, 1954).

V. I. Burenkov (1968), "Imbedding and continuation for classes of differentiable functions of several variables defined in the whole space", in: Progress in Mathematics, Vol. 2: Mathematical Analysis, pp. 73-161 = Engl. trans. of Itogi Nauki-Seriya Matematika (Russian) (Plenum Press, New York, 1968).

P. L. Butzer and R. J. Nessel (1971), Fourier Analysis and Approximation, Vol. I: Onedimensional Theory (Birkhäuser, Basel, and Academic Press, New York, 1971).

A. Friedman (1963), Generalized Functions and Partial Differential Equations (Prentice Hall, Englewood Cliffs, N.J., 1963).

M. I. Ganzburg (1975), "Some inequalities for polynomials in symmetric spaces", Abstracts of Scientific Communications, Conference on the Theory of Approximation of Functions, Kalouga, 1975, pp. 32-33 (Russian).

E. Görlich and G. Paulus (to appear), "A review on Bernstein-type inequalities".

I. I. Ibragimov (1958), "Extremal problems in the class of trigonometric polynomials", Dokl. Akad. Nauk SSSR 121, 415-417 (Russian).

I. I. Ibragimov (1959), "Extremal problems in a class of integral functions of finite degree", Izv. Akad. Nauk SSSR Ser. Mat. 23, 243-256 (Russian). 
I. I. Ibragimov (1965), "Certain extremal problems in the class of entire functions of finite degree", in: Studies Contemporary Problems Constructive Theory of Functions, pp. 212-219 (Russian) (Izdat. Akad. Nauk Azerbaidzan SSSR, Baku, 1965).

I. I. Ibragimov and A. S. Dzafarov (1961), "On some inequalities for an entire function of finite degree and its derivatives", Soviet Math. Dokl. 2, 699-702 = Engl. transl. of Dokl. Akad. Nauk SSSR 138, 755-758 (Russian).

I. I. Ibragimov and D. I. Mamedhanov (1964), "Connection between weighted norms of an entire function of finite degree on lines parallel to the real axis", Soviet Math. Dokl. 5, 917-920 = Engl. transl. of Dokl. Akad. Nauk SSSR 157, 258-261 (Russian).

I. I. Ibragimov and B. A. Rymarenko (1966), "Some extremal problems with side conditions for the class of entire functions of finite degree", Soviet Math. Dokl. 7, 63-65 = Engl. transl. of Dokl. Akad. Nauk SSSR 166, 278-280 (Russian).

D. Jackson (1933), "Certain problems of closest approximation", Bull. Amer. Math. Soc. 39, 890-906.

G. K. Lebed (1957), "Inequalities for polynomials and their derivatives", Dokl. Akad. Nauk SSSR 117, 570-572 (Russian).

D. I. Mamedhanov (1964), "Inequalities for positive entire functions in a generalized Lebesgue space”, Soviet Math. Dokl. 5, 977-980 = Engl. transl. of Dokl. Akad. Nauk SSSR 157, 526-528 (Russian).

D. I. Mamedhanov (1974), "Inequalities of S. M. Nikolskii type for polynomials in a complex variable on curves", Soviet Math. Dokl. 15, 34-37 = Engl. transl. of Dokl. Akad. Nauk SSSR 214, 37-39 (Russian).

H. J. Mertens, R. J. Nessel, and G. Wilmes (1976), "Uber Multiplikatoren zwischen verschiedenen Banach-Räumen im Zusammenhang mit diskreten Orthogonalentwicklungen", Forschungsberichte des Landes Nordrhein-Westfalen 2599, 1-55 (Westdeutscher Verlag, Opladen).

R. J. Nessel and G. Wilmes (1976), "On Nikolskii-type inequalities for orthogonal expansions", in: Approximation Theory II, 479-484 (Academic Press, New York, 1976).

R. J. Nessel and G. Wilmes (to appear), "Inequalities of Bernstein-Nikolskii-type for regular spectral measures".

S. M. Nikolskii (1951), "Inequalities for entire functions of finite degree and their application to the theory of differentiable functions of several variables", Amer. Math. Soc. Transl. Ser. 2, 80 (1969), 1-38 = Engl. transl. of Trudy Mat. Inst. Steklov 38, 244-278 (Russian).

S. M. Nikolskii (1974), Approximation of Functions of Several Variables and Embedding Theorems (Springer-Verlag, Berlin, 1974).

N. M. Sabziev (1965), "An extremal problem in the class of trigonometric polynomials", in: Studies Contemporary Problems Constructive Theory of Functions, pp. 265-272 (Russian) (Izdat. Akad. Nauk Azerbaidzan SSSR, Baku, 1965).

L. Schwartz (1966), Théorie des Distributions (Hermann, Paris, 1966).

E. M. Stein and G. Weiss (1971), Introduction to Fourier Analysis on Euclidean Spaces (Princeton University Press, Princeton, 1971).

I. Szalay (1975), "On the best approximation of factorized Fourier series", in: Approximation Theory, pp. 235-241 (Polish Scientific Publishers, Warszawa-Dordrecht, 1975).

G. Szegö and A. Zygmund (1953), "On certain mean values of polynomials", J. Analyse Math. $3,225-244$.

A. F. Timan (1963), Theory of Approximation of Functions of a Real Variable (Macmillan, New York, 1963).

M. F. Timan (1975), "On a property of orthonormed systems satisfying an inequality of S. M. Nikolskii ", Abstracts of Scientific Communications, Conference on the Theory of Approximation of Functions, Kalouga, 1975, pp. 101-102 (Russian).

F. Treves (1967), Topological Vector Spaces, Distributions and Kernels (Academic Press, New York, 1967). 
H. Triebel (1977), "General function spaces, II: Inequalities of Plancherel-Polya-Nikolskiitype, $L_{p}$-spaces of analytic functions: $0<p \leqslant \infty$ ", J. Approximation Theory 19, 154-175.

C. Watari and Y. Okuyama (1975), "Approximation property of functions and absolute convergence of Fourier series", Tóhoku Math. J. 27, 129-134.

A. Zygmund (1959), Trigonometric Series I, II (Cambridge University Press, Cambridge,1959).

Technical University of Aachen

Aachen 51, Templergraben 55

Federal Republic Germany 